\title{
Nutrition, microbiome, aging, and cancer
}

\begin{abstract}
The research aims at linking cancer with age and other biological and dietary factors. According to Surveillance, Epidemiology, and End Results (SEER) data, aging is one of the critical cancer risk factors for both sexes. The research employed a stringent approach that confirmed the hypothesis linking acquisition of cancerous infections with age. Precisely, individuals are more susceptible to cancerous infections at the latter stages of their lifecycle. The study utilized qualitative approach to gather relevant information and findings were derived from secondary sources such as books, journals, and credible websites. An in-depth acquired knowledge was presented in a narrative form to address the research questions developed from the research objectives. While cancer is a multifactorial disease of the human body, two underlying mechanisms were commonly identified in elderly from the literature. The fundamental mechanisms are low chronic inflammation and immunosenescence. Microbiota, mainly probiotics, could potentially influence the two mechanisms directly and via microbially produced metabolites. Additionally, dietary interventions have the potential to modify microbiota and the inflammatory status. The phenomenon influences the risk and progression of some chronic diseases including cancer. The challenging factors are the identification of the subgroups who will benefit from the initiatives, the time and duration of each nutrition intervention, and early detection of any undesirable effects of those interventions.
\end{abstract}

Volume 7 Issue 3 - 2019

\author{
Faiza Ahmed,' Tony L Brown, ${ }^{2}$ Gabriela \\ Riscuta, ${ }^{2}$ Dan Xi PhD, ${ }^{2}$ Anna Zillow ${ }^{2}$ \\ 'Avalon University School of Medicine, Curacao \\ ${ }^{2}$ National Cancer Institute, National Institutes of Health, USA
}

Correspondence: Tony L Brown, National Cancer Institute, National Institutes of Health, USA, Tel 267-973-6I56,

Email tbrown@post.harvard.edu

Received: June 01, 2017 | Published: June 10,2019

\section{Introduction}

Currently, cancer is regarded to be a menace in the society due to the severity of its impacts, and the mortality rate associated with it. ${ }^{1}$ It represents the second leading cause of death just below cardiovascular ailments; however, in recent times, the mortality rate affiliated to cancer has been declining due to early diagnosis, detection, and treatment of cancerous growth. In this perspective, the progressive increase in life expectancy has a significant correlation with the steady increase in cancer acquisition risk. As such, cancer and age share a lot in common rather than antagonistic features. In the modern context, older individuals and the youth are susceptible to cancer infections. ${ }^{1}$ Precisely, according to the author, a majority of the cancer cases are diagnosed after the age of 65 years. Other instrumental aspects, such as lifestyle choices should be taken into consideration while determining the variants that stimulate cancer in the society. According to Bentley, Brown, Garcia \& Dregansky ${ }^{2}$ inflammation caused by certain dietary elements and biological factors is the sole trigger of cancer acquisition. The inflammatory effects also vary depending the recipient's age, with the older individuals more susceptible to the condition. A significant number of epidemiological studies have established a strong relationship between nutrition and acquisition of core human cancers, such as breast cancer and prostate cancer. As such, it would be viable to deduce that certain dietary components could either inhibit or promote cancerous growths. In particular, bioactive food elements, such as retinoid and selenium, have the potential to suppress cancer.

\section{Objectives}

The objectives will provide the general goal to be attained at the end of the study. The core purpose of this study is to establish the correlation between age, nutrition, biological components, and cancer in the modern context. As such, the study aims to indicate how various nutritional elements and microbial activities affect cancer among the elderly people. The specific objectives for the study are indicated below:
I. To examine the association between diet and dietary components, microbiota composition, and inflammatory status in health and disease of the elderly.

II. Investigate how changes in the microbiota influence the inflammatory and immune status with age.

III. Evaluate the relationship between inflammation and cancer.

\section{Methods}

This section indicates the outline and approaches utilized in the aforementioned objectives. The study deployed qualitative approach to derive fundamental information that would aid in answering the research questions. Specifically, the study utilized exploratory research design since the phenomenon under consideration was new and had limited prior researches. The research further used narrative review (descriptive research) to acquire and present relevant information from diversified sources. Data was collected from authentic and credible secondary sources, such as books, journals, and reliable websites in relation to the study variables specified in the topic. Additionally, to the relevancy to the topic under consideration, the secondary sources were peer-reviewed, less than five years old, and done within the context of the United States and United Kingdom. Moreover, the method employed a meta-analysis of the results; therefore, aligning the findings in a chronological and summarized pattern. The crucial data were obtained via maximizing on the virtual search engines, such as Google Scholar and Science Direct database. The aforementioned databases were favorable for the research due to the magnitude of relevant resources available, efficiency, and limited restrictions therein. A total of 30 articles were reviewed, and 11 met the threshold to be utilized in conducting the research. The rest were discarded due to their inefficiency and inadequacies to meet the requisite standards.

\section{Results}

From the literature review, crucial information pertaining the correlation between age, nutrition, biological factors, and cancer were obtained and incorporated in the research findings. Mentioned below 
are some of the critical findings derived from the secondary sources that would assist in the achievement of the set research questions.

The first finding indicates that aging leads to the reduction of microbiota diversity associated with increased health risk. Studies on aging gut microbiota revealed that the composition of microbiota in elders is considerably different relative to the youths. ${ }^{3}$ A minute level of phylum Firmicutes and an overall low diversity were detected in elderly subjects contrary to the youths. Low diversity has been strongly associated with increased incidences of health risks. ${ }^{3}$ Fecal samples from volunteers with high and low frailty scores indicated a significant reduction in the number of lactobacilli. ${ }^{4}$ At much higher population levels, both the Bacteroides/Prevotella and the Faecalibacterium prausinitzii group showed a significant reduction in the percentage of the total number of hybridizable bacteria in the elderly with high frailty scores versus low frailty scores.

The second finding indicated that age-related microbiota dynamism influences inflammation within humans. Higher than average levels of age-associated inflammation are strong predictors of overall ill health, development of chronic inflammatory conditions, and all-cause mortality in the elderly. ${ }^{5}$ In addition, the levels of pro-inflammatory cytokines, such as IL6, and tumor necrosis factor in the circulation and tissues increases with age in both humans and mice. ${ }^{6,7}$ The presence of a compromised microbiota in centenarians is associated with an even more increased inflammatory status as determined by a range of peripheral blood inflammatory markers. ${ }^{3}$

The third finding indicated that anti-biotic related microbiota reduction promotes inflammation in the human anatomy. An association between altered microbiota and mucosal inflammation was suggested when patients who had recently taken anti-biotics suffered from irritable bowel syndrome symptomatology. ${ }^{5}$ Predictably, antibiotic treatment in the elderly has been reported to affect both richness and diversity of the microbiota and is associated with decrease in bifidobacterial, as well as Bacteroides-Prevotella group. ${ }^{6,7}$

The fourth finding indicated that inflammation precedes cancer in numerous occasions. Inflammation precedes cancer development in several pathophysiological mechanisms such as infections with Helicobacter pylori, a bacterium found in approximately $66 \%$ of the humans ${ }^{8}$ and a cause of global cancer mortality that works by triggering host's inflammatory response. ${ }^{9}$ The hepatic inflammation, which is influenced by gastrointestinal microbiota, predisposes to hepatocellular carcinoma, which is the third most cause of cancer mortality globally. ${ }^{10}$ Additionally, according to Bentley et al., ${ }^{2}$ \& Grivennikov ${ }^{11}$ the mechanism of irritable bowel disease involves bacterial-driven inflammation that promotes colon cancer.

The fifth finding indicated that diet and dietary components influence inflammatory status in humans. Dietary components contribute in determining gut microbiota composition and diversity. ${ }^{12}$ The aforementioned effects involve reduction in LGI due to altered gut microbiota, or they may involve other aspects of microbiota host crosstalk among many other dietary components that might affect inflammation directly. A systematic review of 52 clinical trials examining correlation between dairy consumption and inflammatory markers extrapolates that fermented products in particular, and dairy generally promote anti-inflammatory cascades. This aspect could be beneficial in certain types of cancers. ${ }^{2}$

\section{Conclusion}

From the above findings, it would be plausible to conclude that inflammation increases with age. In addition, it would be viable to deduce that inflammation is a core risk factor for developing cancer, but the mechanisms are not entirely understood. Gut microbiota is one important factor in age associated inflammation and cancer. Aged microbiota may trigger inflammation and impair immune function. Inflammation has been linked to both microbiota and cancer. Microbiota can influence cancer not only by constituent activation of inflammation cascades, but also by triggering genotoxic pathways and tumor microenvironment perturbation. Our study suggests that nutritional habits, such as prebiotics, foods containing probiotics and whole foods, can manifest a net increase of anti-inflammatory markers in the body.

\section{Acknowledgments}

None.

\section{Conflicts of interest}

Author declares that there is no conflict of interest.

\section{References}

1. Zinger A, Cho WC, Ben-Yehuda A. Cancer and aging-the inflammatory connection. Aging and disease. 2017;8(5):611.

2. Bentley R, Brown TL, Garcia E, et al. The role of probiotics in microbial resistance treatment and why they should be added to the World Health Organization's List of Essential Medicines. Int J Community Fam Med. 2018;2:13.

3. Kumar M, Babaei P, Ji B, et al. Human gut microbiota and healthy aging: recent developments and future prospective. Nutrition and Healthy Aging. 2016;4(1):3-16.

4. Van Tongeren SP, Slaets JP, Harmsen, HJM, et al. Fecal microbiota composition and frailty. Applied and environmental microbiology. 2005;1(10):6438-6442.

5. Thevaranjan N, Puchta A, Schulz C, et al. Age-associated microbial dysbiosis promotes intestinal permeability, systemic inflammation, and macrophage dysfunction. Cell host \& microbe. 2017;21(4):455-466.

6. Myriam N Bouchlaka, Gail D Sckisel, Mingyi Chen, et al. Aging predisposes to acute inflammatory induced pathology after tumor immunotherapy. JEM. 2013;210(11):2223.

7. Franceschi C, Capri M, Monti D, et al. Inflammaging and antiinflammaging: a systemic perspective on aging and longevity emerged from studies in humans. Mech Ageing Dev. 2007;128(1):92-105.

8. Helicobacter pylori and Cancer. 2013.

9. Suzuki M, Mimuro H, Kiga K, et al. Helicobacter pylori CagA phosphorylation-independent function in epithelial proliferation and inflammation. Cell host \& microbe. 2009;5(1):23-34.

10. Dapito DH, Mencin A, Gwak GY, et al. Promotion of hepatocellular carcinoma by the intestinal microbiota and TLR4. Cancer cell. 2012;21(4):504-516.

11. Grivennikov SI. Inflammation and colorectal cancer: colitis-associated neoplasia. Seminars in immunopathology. 2013;35(2):229-244.

12. Calder PC, Bosco N, Bourdet-Sicard R, et al. Health relevance of the modification of low-grade inflammation in aging (inflammaging) and the role of nutrition. Aging Res Rev. 2017;40:95-119. 